\title{
ESTUDIO PSICOMÉTRICO SOBRE EL EXAMEN DE ADMISIÓN 2008-I A LA UNIVERSIDAD NACIONAL MAYOR DE SAN MARCOS
}

\author{
Carlos Ponce D. ${ }^{1}$, Miguel Escurra M. ${ }^{2}$ \\ Universidad Nacional Mayor de San Marcos, Perú \\ (RECIBIDO 01/02/2008, ACEPTADO 15/05/2008)
}

\begin{abstract}
RESUMEN
En el presente estudio exploratorio de tipo descriptivo, se plantea la siguiente pregunta de investigación: ¿El examen de admisión 2008-I a la Universidad Nacional Mayor de San Marcos cumple con los requisitos psicométricos exigidos para trabajos de esta naturaleza? El objetivo general de la investigación ha sido: Diagnosticar las características psicométricas básicas que ha presentado el examen de admisión 2008-I a la Universidad Nacional Mayor de San Marcos, es decir: la confiabilidad de la prueba, la validez de los ítemes y el análisis de ítemes (índice de dificultad, poder discriminativo y eficiencia de las respuestas de distracción), respectivamente.
\end{abstract}

El diseño de investigación corresponde al descriptivo simple y nos ha permitido analizar desde la perspectiva psicométrica los resultados obtenidos por los 18588 postulantes al proceso de admisión 2008-I.

El instrumento utilizado ha sido la prueba de admisión 2008-I a la UNMSM, que comprendió un total de 100 ítemes binarios o dicotómicos de tipo opción múltiple, cinco alternativas. De ese total, la mitad del examen, es decir, 50 preguntas corresponden al área de habilidades que tiene dos partes: verbal, compuesta por 25 elementos y numérica que comprende los otros 25 ítemes.

La otra parte de la prueba, compuesta por 25 ítemes, comprende diversas áreas del conocimiento y es elaborada y aplicada indistintamente en relación a las diferentes especialidades que ofrece la Universidad Nacional Mayor de San Marcos.

El análisis estadístico se llevó a cabo teniendo en cuenta un análisis descriptivo y exploratorio para describir de manera detallada el análisis psicométrico del examen de admisión. En la prueba de admisión 2008-I a la UNMSM, los resultados se aproximan a la distribución normal, por lo que ha sido posible trabajar con estadísticos paramétricos. Los resultados corroboran que las preguntas del examen son consistentes entre sí. El análisis de la confiabilidad por consistencia interna nos indica coeficientes alfa razonables en las seis áreas examinadas, lo que nos permite inferir la existencia de puntajes confiables.

Entre las conclusiones más importantes del presente estudio tenemos: 1) La prueba de admisión 2008-I en su conjunto ha presentado una confiabilidad aceptable en las dos partes

1 Docente Principal de la Facultad de Psicología de la Universidad Nacional Mayor de San Marcos.

E-mail: cponcediaz@hotmail.com

2 Docente Principal de la Facultad de Psicología de la Universidad Nacional Mayor de San Marcos. 
que la componen: habilidad y conocimientos. 2) El análisis de la validez de los ítemes que conforman la prueba son indicadores de que el examen mide en su conjunto lo que pretende medir. 3) Los grados de dificultad de los ítemes se concentraron en los niveles intermedio y difíciles, encontrándose un reducido grupo de preguntas estimadas fáciles. 4) El análisis de ítemes revela que las preguntas del examen han presentado mayoritariamente una discriminación positiva, es decir, los postulantes con mayores puntajes en la prueba total acertaron en los ítemes más frecuentemente que los que lograron los menores calificativos en el examen total. 5) En líneas generales, se puede afirmar que la prueba de admisión 2008-I ha cumplido satisfactoriamente con los requisitos psicométricos exigidos para exámenes de esta naturaleza.

Palabras clave: Psicometría, Validez, Confiabilidad, Ítemes, Ítem Binario.

\begin{abstract}
In the subsequent exploratory descriptive study, we formulated the following investigation question: Does the 2008-I admission exam of the Major National University of San Marcos, cover all the psychometric requirements demanded by an evaluation of that kind of nature? The General Objective of the investigation is to: Identify the basic psychometric characteristics which the 2008-I admission exam of the Major National University of San Marcos has presented, consisting in the reliability of the test, the validity of the items and their analysis.
\end{abstract}

The investigational design corresponds to the simple descriptive form and has permitted us to analyze the psychometric perspective of the results obtain from 18,588 postulants in the 2008-I admissions process.

The instrument utilized in the investigation is the 2008-I admission exam of the UNMSM, which consisted of a total of 100 binary and dichotomic items in multiple choice form, consisting in five alternatives. Half of the Items of the exam, that is 50 questions, correspond to the area of verbal and numeric abilities, each subarea consisting of 25 items. The other part of the test is composed by 50 items that of which corresponds to the different areas of knowledge which have a direct relation to the different study departments of the Major National University of San Marcos has to offer.

The statistic process was done taking into account the descriptive and exploratory analysis to describe in a detailed manner the psychometric analysis of the admission exam. The results obtained from the investigation of the 2008-1 process of admission of the UNMSM showed a normal distribution, permitting us to utilize parametric statistics. The results corroborate the consistency between the questions employed by the exam. The internal consistency from the reliability analysis indicate reasonable alpha coefficients in all six areas that were examined, which grants us permission to inferior the existence of reliable results.

Amongst the most important conclusions stemming from the following study we can mention: 1) The 2008-I admission exam, en its entirety has presented an acceptable level of reliability en both areas it is comprised of, which are abilities and knowledge. 2) The validity analysis of the items which make up the test, indicate that the exam measures en its entirety what it iis set out to measure. 3) The different levels of difficulty in the items are concentrated in the intermediate and hard levels, leaving a small group of questions which are estimated as easy. 4) The item analysis reveals that the questions from the exam have presented in its majority a positive discrimination, that is that the postulants with higher scores on the entire exam answered correctly to more items that of students who obtained lower scores on the 
exam. 5) In general, one can affirm that the 2008-I admission exam has satisfactory complied with the psychometric requirements necessary for an exam of this nature.

Keywords: Psychometrics, Validity, Reliability, Items, Binary Items.

\section{INTRODUCCIÓN}

Los estudios contemporáneos en psicometría revelan que las diferencias individuales existen, son medibles y constituyen una temática importante en diversas áreas de la psicología aplicada, especialmente en la educacional y en donde la temática de la selección para el ingreso a la Universidad, constituye una tarea que debe ser resuelta de la mejor manera por las instituciones académicas, toda vez que garantiza la incorporación del recurso humano más idóneo para desarrollarse en la vida académica y profesional.

Precisamente, es de conocimiento de la opinión pública que a nuestra Cuatricentenaria Casa de Estudios postulan todos los años miles de estudiantes que compiten por alcanzar alguna de las vacantes en las especialidades a concurso, motivados a estudiar en virtud al prestigio y al nivel de enseñanza de San Marcos.

Independientemente de las capacidades y de la performance que tenga cada uno de los postulantes a nuestra Universidad, creemos que una tarea fundamental que corresponde a nuestra Institución es garantizar que el examen de admisión, construido y aplicado para seleccionar al material humano, cumpla satisfactoriamente los requisitos psicométricos básicos exigidos para pruebas de esta naturaleza.

De allí que en el presente estudio exploratorio de tipo descriptivo, nos planteamos la siguiente pregunta de investigación: ¿El examen de admisión 2008-I a la Universidad Nacional Mayor de San Marcos, cumple con los requisitos psicométricos exigidos para trabajos de esta naturaleza?

\section{Justificación}

El análisis psicométrico de una prueba objetiva del tipo selección para el ingreso a la Universidad constituye una temática muy importante en el mundo académico y ha sido poco estudiada en las universidades de nuestro País, especialmente en San Marcos.

El conocimiento de esta temática nos va a permitir lo siguiente:

a) Que los docentes elaboradores de las preguntas y de los diversos tipos de prueba conozcan objetivamente las características psicométricas del producto elaborado una vez aplicado.

b) Que los postulantes tengan conocimiento de que el Examen de Admisión no queda en la simple administración y entrega de resultados, sino que en base a los resultados obtenidos se están investigando permanentemente los hallazgos a fin de optimizar la elaboración de nuevas pruebas.

c) Que la confianza que la opinión pública deposita en San Marcos, en materia de examen de admisión, tiene como garantía la seriedad y responsabilidad con que los 
ESTUDIO PSICOMÉTRICO SOBRE EL EXAMEN DE ADMISIÓN 2008-I A LA UNMSM

diversos equipos de docentes de la Oficina Central de Admisión (OCA), encargados de elaborar y perfeccionar nuestro examen, cumplen con sus obligaciones

\section{Objetivo}

Diagnosticar las características psicométricas que ha presentado el examen de admisión 2008-I a la Universidad Nacional Mayor de San Marcos; es decir, la confiabilidad de la prueba; la validez de los ítems y el análisis de ítemes (índice de dificultad, poder discriminativo y eficiencia de las respuestas de distracción), respectivamente.

\section{MÉTODO}

\section{El diseño de investigación}

Corresponde al descriptivo simple y permite analizar las características psicométricas del examen de admisión 2008-I.

El número de inscritos a dicho proceso fue de 19099 postulantes para cubrir 1807 vacantes en las 59 carreras que ofrecen las 20 Facultades de la UNMSM.

\section{La muestra}

Estuvo conformada por 18588 postulantes de ambos sexos (9460 mujeres y 9128 varones), provenientes de Colegios estatales (12 005) y Particulares (6583), de diversos departamentos y provincias de nuestro país, especialmente de Lima.

\section{Instrumento}

La prueba de admisión 2008-I a la Universidad Nacional Mayor de San Marcos comprendió un total de 100 ítemes binarios o dicotómicos del tipo opción múltiple, cinco alternativas.

De ese total, 50 preguntas corresponden al área de habilidades, que tiene una parte Verbal compuesta por 25 reactivos, que a su vez comprende dos subáreas: Comprensión de Lectura: 15 ítemes y Usos del Lenguaje: 10 ítemes, respectivamente. La otra parte es Numérica compuesta por 25 preguntas y comprende a su vez cinco subáreas: aritmética, álgebra, geometría, trigonometría y razonamiento lógico, conformada por cinco reactivos cada una. Esta parte del Examen de Admisión es aplicada a todos los postulantes a la Universidad.

Las otras 50 preguntas son de conocimientos y comprende las áreas de: lenguaje, matemáticas, física, química, biología, economía, historia, literatura, psicología, filosofía y geografía y para las cuales se construyen 10 preguntas, respectivamente.

Esta parte del Examen se elabora y se aplica indistintamente en relación a las áreas de especialidad que ofrece la Universidad de San Marcos. 


\section{Confiabilidad}

La confiabilidad de un test se refiere a la consistencia o precisión de las puntuaciones obtenidas por los mismos individuos cuando son examinados con la misma prueba; en una sola administración del test o en diferentes ocasiones o cuando son examinados con conjuntos distintos de preguntas equivalentes o bajo otras condiciones variables de examen.

En su sentido más amplio, indica hasta qué punto pueden atribuirse a errores de medida las diferencias individuales en las puntuaciones del test y hasta qué punto pueden deberse a diferencias verdaderas en las características o dimensión que estamos sometiendo a medición.

En nuestra investigación, para su obtención, hemos utilizado la fórmula KR20 de KuderRichardson, que para su aplicación requiere tres tipos de información: a) Número de ítemes del test; b) La media o promedio aritmético, y c) La desviación estándar. Técnicamente, la completa ausencia de confiabilidad estaría indicada por un coeficiente de 0.00 y la confiabilidad positiva perfecta por 1.00 .

Por otra parte, cuando se utilizan pruebas tipo salón de clase (que es nuestro caso), los coeficientes de confiabilidad aceptables oscilan entre 0.60 y 0.80 , respectivamente.

\section{Validez}

La validez es un concepto que tiene muchas facetas. Su establecimiento se enmarca dentro de un proceso. En una primera aproximación, puede significar "el grado en que una prueba mide lo que intenta medir".

En todo proceso de validación, la validez de un test se expresa generalmente por medio de un coeficiente de correlación entre sus puntajes y los llamados criterios y, precisamente, la correlación entre los puntajes del test y las medidas de criterio se denomina coeficiente de validez.

En nuestro estudio se ha considerado esta normatividad para analizar la validez de los ítemes del Examen de Admisión 2008-I y se ha utilizado la fórmula de la correlación item-test de Pearson para calcular los valores correspondientes.

\section{Análisis de Ítemes}

Para analizar las preguntas del examen, se utilizó el sistema simplificado de Gronlund (1974), que posibilitó estudiar las respuestas dadas por los postulantes a cada ítem, logrando una información sobre dos aspectos: la dificultad del ítem y el poder discriminativo de cada ítem.

a) La dificultad del ítem, indica si el ítem fue fácil, difícil o intermedio, de acuerdo a las características de su elaboración, y se obtiene determinando el porcentaje de postulantes que respondieron correctamente al ítem, por lo que un porcentaje menor indica mayor dificultad del ítem y viceversa. 
b) El poder discriminativo del ítem, nos indica de qué manera la pregunta discriminó entre los postulantes con puntuaciones altas en el total del Examen, respecto de los que obtuvieron puntuaciones bajas en el test. Su cálculo es de mayor trascendencia y se obtiene comparando el número de probandos de los grupos superior e inferior en la prueba que respondieron correctamente a la pregunta.

Técnicamente, se encontró tres tipos de resultados en materia de poder discriminativo del ítem: máximo poder discriminativo positivo; poder discriminativo positivo; poder discriminativo negativo y poder discriminativo nulo. Las dos primeras modalidades son aceptables y las dos segundas implican la falta de discriminación y por lo tanto la eliminación del ítem.

El caso es que, en líneas generales, el análisis de Ítemes proporciona datos que ayudan a percibir fallas técnicas específicas y con ello a obtener mayor información para el mejoramiento de las preguntas. Inclusive, aún en el supuesto de que no se vaya a usar nuevamente las preguntas (que es nuestro caso), el análisis tiene sus mayores ventajas cuando se diseñan talleres de construcción de ítemes a fin de que exista el equilibrio deseable en lo referente a la dificultad de los ítemes y a la discriminación.

En este punto hay que señalar que la data de los resultados ha permitido considerar dentro del análisis de ítemes lo referente a las respuestas de distracción, distractores, distrayentes u opciones incorrectas, a fin de detectar errores y conceptos equivocados comunes que pueden implicar una ayuda para introducir correcciones en la construcción de las preguntas.

\section{Técnicas de procesamiento y análisis de datos}

El análisis estadístico ha comprendido los siguientes aspectos

Análisis descriptivo y exploratorio para describir de manera detallada la distribución de la muestra examinada; en tal sentido, se han calculado para las variables estudiadas: las frecuencias, los porcentajes, las medias o promedios aritméticos, las desviaciones estándar, así como los análisis de tallos y hojas y las pruebas de ajuste a la curva normal de Kolmogorov-Smirnov para los puntajes de las escalas de las pruebas.

Análisis psicométrico del Examen de Admisión 2008-I, que incluye: un estudio de la confiabilidad a través de la fórmula KR20 de Kuder-Richardson; un estudio de la validez mediante la aplicación de la fórmula de la correlación ítem-test de Pearson y un estudio de la dificultad de los ítemes: indice de dificultad y poder discriminativo de los ítemes mediante el procedimiento simplificado de Gronlund. 


\section{RESULTADOS}

Tabla N. ${ }^{0}$ 1. Estudio de la confiabilidad de la prueba de Admisión 2008-I.

\begin{tabular}{lcc}
\hline \multicolumn{1}{c}{ Áreas del examen } & Confiabilidad & Confiabilidad total \\
\hline 1. HABILIDAD & & \\
1.1 Habilidad Verbal: & & $\mathrm{CL}+\mathrm{UL}=0.73$ \\
- Comprensión de lectura (CL) & 0.69 & \\
- Usos del lenguaje (UL) & 0.52 & \\
\hline 1.2 Habilidad numérica: & & \\
- Aritmética (AR) & 0.50 & \\
- Álgebra (AL) & 0.52 & $\mathrm{AR}+\mathrm{AL}+\mathrm{GE}+$ \\
- Geometría (GE) & 0.60 & $\mathrm{TR}+\mathrm{RL}=0.79$ \\
- Trigonometría (TR) & 0.52 & \\
- Razonamiento Lógico (RL) & 0.55 & \\
\hline
\end{tabular}

\section{CONOCIMIENTO}

- Lenguaje (LE)

- Matemáticas (MA)

- Física (FI)

- Química (QU)

- Biología (BI)

- Economía (EC)

- Historia (HI)

- Literatura (LI)

- Psicología (PS)

- Filosofía (FI)

- Geografía (GE)
0.75
0.68
0.79
0.67
0.65
0.71
0.42
0.64
0.45
0.63
0.61

En la Tabla N. ${ }^{\circ}$ 1, se puede apreciar de manera global que los valores relativos a la confiabilidad son adecuados tanto en el área de habilidad como en el área de conocimientos. La parte de habilidad verbal tiene una confiabilidad total de 0.73 que puede considerarse dentro de los niveles aceptables. En el desagregado de la parte de habilidad verbal, detectamos que la subparte de Comprensión de lectura (CL) alcanza una consistencia de 0.69 , en tanto que la de Usos del lenguaje (UL) obtiene 0.52 , respectivamente.

En lo referente a la habilidad numérica, ésta alcanza una confiabilidad total de 0.79 , es decir, dentro de los niveles aceptables.

En el desagregado de la parte de habilidad numérica, encontramos que las subpartes, es decir Aritmética (AR), Álgebra (AL), Geometría (GE), Trigonometría (TR) y Razonamiento Lógico (RL), alcanzan una consistencia de: $0.50,0.52,0.60,0.52$ y 0.55 , respectivamente. 
En el área de Conocimientos, se ha realizado un estudio de la confiabilidad sobre los 50 ítemes que conforman cada una de las seis áreas de especialidad, detectándose que en el área A-Ciencias de la Salud se registró una consistencia de 0.88; en el área B-Humanidades, 0.82; en el área C-Ciencias Sociales, 0.83; en el área D-Ciencias Básicas, 0.85; en el área E-Ingenierías, 0.86 y en el área F-Económico-Empresariales, 0.81, respectivamente. Por lo tanto, todos son valores adecuados.

En el desagregado del área de Conocimientos, encontramos que las áreas de lenguaje (LE), Matemáticas (MA), Física (FI), Química (QU), Biología (BI), Economía (EC), Literatura (LI), Filosofía (FI) y Geografía (FI), presentan confiabilidades que oscilan entre 0.61 (GE) y $0.79(\mathrm{FI})$, respectivamente.

Por otra parte, en el área de Conocimientos se ha detectado que las áreas de Psicología (PS) e Historia (HI) presentan una confiabilidad de 0.45 y 0.42 , es decir, por debajo de 0.60 .

Tabla N. ${ }^{\circ}$ 2. Validez de los ítemes del Examen de Admisión 2008-I.

\begin{tabular}{|c|c|c|}
\hline \multicolumn{3}{|c|}{ Validez de los ítemes } \\
\hline Áreas del examen & Positivo (+) & Negativo (-) \\
\hline I. Habilidad & Preguntas & Preguntas \\
\hline $\begin{array}{l}\text { 1.1 Habilidad Verbal } \\
\text { - Comprensión de Lectura (CL) } \\
\text { - Usos del Lenguaje (UL) }\end{array}$ & $\begin{array}{l}9-13-5-10-11-3 \\
1-4-2-3-9-5\end{array}$ & $\begin{array}{l}1-2-4 \\
7-6-8-10\end{array}$ \\
\hline $\begin{array}{l}\text { 1.2 Habilidad Numérica } \\
\text { - Aritmética (AR) } \\
\text { - Álgebra (AL) } \\
\text { - Geometría (GE) } \\
\text { - Trigonometría (TR) } \\
\text { - Razonamiento Lógico (RL) }\end{array}$ & $\begin{array}{l}2-3 \\
2-3-4 \\
1-3-2 \\
3-4-1 \\
3-5-1\end{array}$ & $\begin{array}{l}5 \\
5 \\
5 \\
- \\
-\end{array}$ \\
\hline \multicolumn{3}{|c|}{ Validez de los ítemes } \\
\hline Áreas del examen & Positivo (+) & Negativo (-) \\
\hline II. Conocimiento & Preguntas & Preguntas \\
\hline $\begin{array}{l}\text { - Lenguaje (LE) } \\
\text { - Matemáticas (MA) } \\
\text { - Física (FI) } \\
\text { - Química (QU) } \\
\text { - Biología (BI) } \\
\text { - Economía (EC) } \\
\text { - Historia (HI) } \\
\text { - Literatura (LI) } \\
\text { - Psicología (PS) } \\
\text { - Filosofía (FI) } \\
\text { - Geografía (GE) }\end{array}$ & $\begin{array}{l}2-5-8-7 \\
10-3-1 \\
10-5-3-9 \\
6-4-3-5 \\
9-2-1 \\
4-2-6 \\
- \\
3-2-6-9 \\
10-7 \\
6-2-10-4 \\
5-8-9-6-7\end{array}$ & $\begin{array}{l}- \\
- \\
- \\
- \\
4-3-8 \\
- \\
4-8-1-9-5-2-10 \\
8-10 \\
5-9-3-4-8-1 \\
9-1-5 \\
10\end{array}$ \\
\hline
\end{tabular}


En la Tabla N. ${ }^{\circ} 2$, podemos apreciar que en la parte de Habilidades encontramos 10 ítemes cuya validez se encuentra por debajo de 0.20 en la correlación ítem-test y, por lo tanto, presentan una validez por debajo de los niveles deseables.

Las 40 preguntas restantes arrojan valores por encima de $0.20 \mathrm{y}$, por lo tanto, presentan una validez positiva.

Debemos precisar que el mayor número de Ítemes con validez no deseable se presenta en el área de Habilidad verbal: 4 ítemes en usos del lenguaje y 3 en comprensión de lectura.

En el área de Habilidad numérica, se encuentra encontramos un número menor de preguntas con validez negativa: una en aritmética, una en álgebra y una en geometría, respectivamente.

En la parte de Conocimientos, encontramos 22 ítemes cuya validez se encuentra por debajo de 0.20 en la correlación ítem-test y por lo tanto presentan una validez no deseable. Las 23 preguntas restantes presentan valores por encima de $0.20 \mathrm{y}$, por lo tanto, presentan validez positiva.

Es importante subrayar que el mayor número de ítemes con validez no deseable se presenta en las áreas de historia (07); psicología (06): biología (03) y filosofía (03).

Por otra parte, no se detecta validez negativa de los ítemes en las áreas de: lenguaje, matemáticas, física, química y economía, respectivamente. 
ESTUDIO PSICOMÉTRICO SOBRE EL EXAMEN DE ADMISIÓN 2008-I A LA UNMSM

Tabla N. ${ }^{\circ}$ 3. Índice de dificultad de los ítemes según habilidades y conocimientos examinados.

\begin{tabular}{|c|c|c|c|c|}
\hline Grado de dificultad áreas & Fácil & Intermedio & Difícil & Total \\
\hline \multicolumn{5}{|l|}{ I. HABILIDADES } \\
\hline \multicolumn{5}{|l|}{ 1.1 Habilidad Verbal } \\
\hline - Comprensión de Lectura (CL) & 2 & 9 & 4 & 15 \\
\hline - Usos del Lenguaje (UL) & 1 & 6 & 3 & 10 \\
\hline Total de Hab. Verbal & 3 & 15 & 7 & 25 \\
\hline \multicolumn{5}{|l|}{ 1.2 Habilidad Numérica } \\
\hline - Aritmética (AR) & - & 2 & 3 & 5 \\
\hline - Álgebra (AL) & - & 2 & 3 & 5 \\
\hline - Geometría (GE) & - & 3 & 2 & 5 \\
\hline - Trigonometría (TR) & - & - & 5 & 5 \\
\hline - Razonamiento Lógico (RL) & - & 4 & 1 & 5 \\
\hline Total de Hab. Numérica & - & 11 & 14 & 25 \\
\hline Total de habilidades & 3 & 26 & 21 & 50 \\
\hline \multicolumn{5}{|l|}{ II. CONOCIMIENTOS } \\
\hline - Física (FI) & 0 & & & \\
\hline - Lenguaje (LE) & 0 & 5 & & \\
\hline - Matemáticas (MA) & 0 & 6 & & \\
\hline - Física (FI) & 0 & 10 & & \\
\hline - Química (QU) & 0 & 2 & & \\
\hline - Biología (BI) & 0 & 7 & & \\
\hline - Economía (EC) & 0 & 7 & & \\
\hline - Historia (HI) & 1 & 6 & & \\
\hline - Literatura (LI) & 0 & 8 & & \\
\hline - Psicología (PS) & 0 & 6 & & \\
\hline $\begin{array}{l}\text { - Filosofía (FI) } \\
\text { - Geografía (GE) }\end{array}$ & 0 & 2 & & \\
\hline 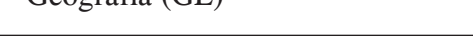 & & & & \\
\hline
\end{tabular}




\section{CONOCIMIENTOS}

\begin{tabular}{|c|c|c|c|c|}
\hline 2.1. Física & 0 & 1 & 9 & 10 \\
\hline 2.2. Química & 0 & 5 & 5 & 10 \\
\hline 2.3. Biología & 0 & 6 & 4 & 10 \\
\hline 2.4. Economía & 0 & 10 & 0 & 10 \\
\hline 2.5. Historia & 0 & 2 & 8 & 10 \\
\hline 2.6. Literatura & 0 & 7 & 3 & 10 \\
\hline 2.7. Psicología & 0 & 7 & 3 & 10 \\
\hline 2.8. Filosofía & 1 & 6 & 3 & 10 \\
\hline 2.9. Geografía & 0 & 8 & 2 & 10 \\
\hline 2.10. Lenguaje & 0 & 6 & 4 & 10 \\
\hline 2.11. Matemáticas & 0 & 2 & 8 & 10 \\
\hline Total de Conocimientos & 1 & 60 & 49 & 110 \\
\hline TAL GENERAL: & 4 & 86 & 70 & 160 \\
\hline
\end{tabular}

En la tabla 3, podemos apreciar que de los 50 ítemes de la parte de habilidades (verbal+numérica), se han detectado -en materia de índice de dificultad-, 3 preguntas fáciles, 26 intermedias y 21 difíciles, respectivamente.

Del mismo modo, se aprecia que de las 11 materias que conforman la parte de conocimientos y que hacen un total de 110 ítemes, se han registrado 1 pregunta fácil, 60 intermedias y 49 difíciles, respectivamente.

El total general nos permite apreciar que de las 160 preguntas existen: 4 fáciles, 86 intermedias y 70 difíciles. 
ESTUDIO PSICOMÉTRICO SOBRE EL EXAMEN DE ADMISIÓN 2008-I A LA UNMSM

Tabla 4. Poder discriminativo o indice de discriminación de las preguntas del Examen de Admisión 2008-I.

ÁREAS DEL EXAMEN

PODER DISCRIMINATIVO DE LOS ÍTEMES

POSITIVO $(+) \quad$ NEGATIVO $(-)$

I. HABILIDAD

PREGUNTAS

PREGUNTAS

1.1. Habilidad verbal

- Comprensión de lectura (CL):

9-10-11-12-14

$1-2$

- Usos del lenguaje (UL):

1-4-3-2-9

7-10

1.2. Habilidad numérica

- Aritmética (AR):

2-4

5

- Álgebra (AL):

$2-1$

5

- Geometría (GE):

1-2-3

5

- Trigonometría (TR):

4-3

$1-2-5$

- Razonamiento lógico (RL):

5-3

II. CONOCIMIENTO

- Lenguaje (LE):

5-2-7

- Matemáticas (MA):

3-1-10

- Física (FI):

5-2

- Química (QU):

6-4-3-2

- Biología (BI):

$9-1-6-2$

- Economía (EC):

- Historia (HI):

7

- Literatura (LI):

2-3-6

- Psicología (PS):

10-7-1-2

- Filosofía (FI):

6-10-2-4

- Geografía (GE):

7-8-5 
En la tabla 4, podemos apreciar en líneas generales que de las 160 preguntas consideradas en el Examen de Admisión 2008-I, 10 ítemes de la parte de habilidades presentan valores por debajo de 0.10 , por lo que revelan una discriminación por debajo de los valores esperados. Las 40 preguntas restantes registran puntajes por encima de $0.10 \mathrm{y}$ por lo tanto poseen discriminación positiva.

En el sub-test de habilidad verbal se detectan 4 preguntas con discriminación negativa (2 en comprensión de lectura y 2 en usos del lenguaje). En el sub-test de habilidad numérica se registran 6 itemes con dicriminación negativa (1 en aritmética, 1 en algebra, 1 en geometría y 3 en trigonometría).

Es importante señalar que en la parte de conocimientos no se detectan itemes con valores por debajo de 0.10 , por lo que todas las preguntas poseen discriminación positiva.

\section{CONCLUSIONES}

1. El Examen de Admisión 2008-I, en su conjunto y como estructura global ha presentado una confiabilidad aceptable en sus dos partes, tanto en lo que corresponde a Habilidad (verbal + numérica), como a Conocimientos. Los hallazgos revelan la consistencia o precisión de las puntuaciones obtenidas por los postulantes a cada una de las áreas de especialidad por lo que las diferencias individuales al momento de elaborar el cuadro de méritos se deben a diferencias verdaderas en las dimensiones que se han sometido a medición. En tal sentido, se debe reconocer a los docentes constructores de preguntas de las diversas temáticas que no obstante hacer sus propuestas por separado, han coincidido en lo referente a la consistencia de la medida.

De manera individual, sin embargo, debemos mencionar que en la parte de habilidad verbal, se debe mejorar lo referente a usos del lenguaje y en habilidad numérica, lo concerniente a aritmética, algebra, geometría, trigonometría y razonamiento lógico, respectivamente.

En la parte de conocimiento algunas áreas como historia y psicología deben mejorar sus propuestas de preguntas toda vez que los valores obtenidos están por debajo de las demás materias.

2. El análisis de la validez de los ítemes del Examen de Admisión 2008-I, en líneas generales, es satisfactorio. En tal sentido hemos detectado que de las 160 preguntas elaboradas( 50 de habilidades y 110 de conocimientos), 128 presentan una validez positiva en tanto que 32 presentan una validez que puede estimarse por debajo de los niveles esperados.

Los hallazgos son indicadores de que la prueba mide en su conjunto lo que pretende medir.

De manera individual, sin embargo, debemos precisar que en la parte de habilidad verbal, se debe mejorar lo referente a la elaboración de los ítemes de comprensión de lectura y usos del lenguaje. 
En la parte de conocimiento, algunas áreas como historia y psicología deben mejorar sus propuestas toda vez que de 10 reactivos hay fallas en 7 y 5 preguntas. En menor medida, se encuentran las áreas de filosofía (3), biología (3), y literatura (2), respectivamente.

3. Del total de 160 ítemes elaborados por los diversos colegas docentes de las diversas facultades para el Examen de Admisión 2008-I, hemos detectado que 4 han obtenido el índice de dificultad de fácil; 86 el de intermedio y 70 el de difícil.

En la práctica, debieron haber un $30 \%$ de fáciles (48); un $40 \%$ de intermedios (64); y un $30 \%$ de difíciles (48), respectivamente.

Los resultados nos indican que no obstante los docentes constructores de ítemes elaboran sus preguntas (en el número y porcentajes esperados), con los tres grados de índice de dificultad mencionados, sin embargo los resultados nos indican de manera específica que casi no hay preguntas fáciles y que en nuestro caso sólo ha habido 4 que corresponden al $2.5 \%$ del total de preguntas. En suma, los grados de dificultad se concentran en los niveles intermedio y difíciles.

Cabe mencionar que de las 4 preguntas con valores fáciles, 3 corresponden a habilidad verbal y 1 a la temática de historia. Por otra parte, las preguntas difíciles en el área de conocimiento se han concentrado en las temáticas de física, matemáticas e historia, respectivamente.

4. Las preguntas del Examen de Admisión 2008-I han presentado mayoritariamente una discriminación positiva, es decir, los postulantes con mayores puntajes en la prueba total acertaron en los ítemes analizados más frecuentemente que los postulantes que obtuvieron los menores puntajes en la prueba total. En otras palabras, 150 de los 160 ítemes están discriminando, cada uno, entre los postulantes de la misma manera que el puntaje total.

Del total de 160 ítemes, 150 registraron una discriminación positiva y solamente 10 presentaron una discriminación negativa.

Los ítemes con discriminación negativa (10), se concentraron en el área de habilidad en tanto que en el área de conocimientos no se registraron reactivos con discriminación negativa, es decir, todos sin excepción tuvieron discriminación positiva.

5. Técnicamente, los resultados obtenidos revelan que, en líneas generales, la prueba de Admisión 2008-I ha cumplido satisfactoriamente con los requisitos psicométricos exigidos en la construcción de exámenes de esta naturaleza: confiabilidad de la prueba, validez de los ítemes y análisis de reactivos (indice de dificultad y poder discriminativo), respectivamente.

Creemos que los hallazgos también constituyen una expresión cuantitativa de la seriedad, responsabilidad e identificación con la que actuaron los docentes de las diversas facultades que estuvieron comprometidos con la elaboración de la prueba, es decir, como un equipo de trabajo dispuesto a los mayores y mejores logros y que hacen que la comunidad nacional perciba a nuestra Cuatricentenaria Universidad como un modelo a seguir. 


\section{SUGERENCIAS}

1. Planificar el estudio psicométrico del próximo examen de Admisión 2008-II, programado para la primera semana de marzo del presente año, incluyendo en la data no sólo la información referente a lo tratado en la presente investigación, sino lo referente a las respuestas de distracción, distractores, distrayentes o respuestas incorrectas, a fin de proceder a un análisis de ítemes más completo que el que se ha efectuado en la presente investigación.

2. Que en los seminarios-taller de elaboración de ítemes se tengan en cuenta aquellas preguntas que han tenido un resultado positivo y que pueden ser usadas como modelos a seguir por parte de los constructores. Del mismo modo, deben tenerse en cuenta aquellos reactivos que han tenido valores negativos y que pueden utilizarse como referencia de lo que no debe hacerse en materia de construcción de ítemes.

3. Elaborar Normas o Baremos (Eneatipos, Decatipos, Percentiles, etc.), que nos permitan entender el nivel y la ubicación del recurso humano que ha ingresado a la Universidad, respecto del total de estudiantes que postularon; en buena cuenta, precisar de qué manera se han incorporado a las diversas áreas de especialidad grupos de estudiantes que acrediten un nivel académico que les permita desarrollarse profesionalmente y competir a satisfacción en el mercado de trabajo.

4. Efectuar un análisis factorial de primer orden e incluso de segundo orden para estudiar en el primer caso si las preguntas que se han elaborado para una determinada temática corresponden efectivamente a dicha temática o se vinculan simultáneamente con otras. En el segundo caso, el análisis estará destinado a precisar si algunas comparaciones o correlaciones que podrían surgir en el estudio son auténticas o ficticias.

5. Los resultados obtenidos abren la posibilidad de efectuar a corto plazo otros estudios que permitan investigar con objetividad la relación entre los resultados del Examen de Admisión y el rendimiento académico de los ingresantes a cada una de las áreas de especialidad

6. Del mismo modo, los hallazgos de la presente investigación deben llevar a los constructores de la prueba de admisión a innovar permanentemente y perfeccionar el producto construido a fin de optimizar los valores psicométricos obtenidos en esta oportunidad.

\section{REFERENCIAS BIBLIOGRÁFICAS}

1. Aiken, L.R. (1985). Tres coeficientes para analizar la confiabilidad y validez de las calificaciones. Educational and Psychological Measurement, 45, 131-142.

2. Anastasi, A. (1981). Capacitación, avance de las pruebas y capacidades desarrolladas. American Psychologist, 36, 1086-1093.

3. Bodner, G.M. (1980). Análisis estadístico de los exámenes de opción múltiple. Journal of Chemical Education, 57, 188-190. 
ESTUDIO PSICOMÉTRICO SOBRE EL EXAMEN DE ADMISIÓN 2008-I A LA UNMSM

4. Brown, J. y Burton, R.R. (1978). Modelos de diagnóstico para defectos de procedimiento en las habilidades matemáticas. Cognition Science, 2, 155-191.

5. Brown, W.R. y McGuire, J.M. (1976). Prácticas actuales de evaluación psicológica. Professional Psychology, 7, 475-484.

6. Carver, R.P. (1974). Dos dimensiones de las pruebas: psicométrica y edumétrica. American Psychologist, 29, 512-518.

7. Eysenck, H.J. (1956). La herencia de la extroversión-introversión. Acta Psicológica, 12. 95-110.

8. Gronlund, Norman E. (1974). Elaboración de tests de aprovechamiento. Editorial Trillas, México, D.R.

8. Hsu, T.C y Moss, P.A. y Khampalikit, C. (1984). Los méritos de los conceptos de respuesta múltiple al evaluarlos con el uso de seis fórmulas de calificación. Journal of Experimental Education, 52, 152-158.

10. Serlin, R.C. y Kaiser, H.F. (1978). Método para incrementar la confiabilidd de la prueba corta de opción múltiple. Educational and Psychological Measurement, 38, 337-340.

11. Snack, W. V. y Porter, D. (1980). La prueba de aptitud académica: una apreciación crítica. Harvard Educational Review, 50, 154-175.

12. Tavella, Nicolás M. (1978). Análisis de ítemes en la construcción de instrumentos psicométricos. Edit. Trillas. México, D.F. 